\title{
Accumulation Ratio AUC to Last Nonzero Concentration
}

National Cancer Institute

\section{Source}

National Cancer Institute. Accumulation Ratio AUC to Last Nonzero Concentration. NCI

Thesaurus. Code C139129.

The area under the curve (AUC) from the time of dosing to the last measurable concentration divided by the area under the curve from the time of dosing to the last measurable concentration during the initial dosing interval. 